\title{
Acknowledgement to Referees for Skin Appendage Disorders 2016
}

Skin

Appendage Disorders

The Editors extend their gratitude and appreciation to the following reviewers whose comments and criticisms ensure the quality of the papers published in this journal.

Roberto Arenas, Tlalpan, Mexico Giuseppe Argenziano, Reggio Emilia, Italy Daniel Asz Sigall, Mexico City, Mexico Robert Baran, Cannes, France Wilma F. Bergfeld, Cleveland, OH, USA Richert Bertrand, Brussels, Belgium Vincenzo Bettoli, Ferrara, Italy Ulrike Blume-Peytavi, Berlin, Germany Anna Campanati, Ancona, Italy Ralph Daniel, Jackson, MS, USA David de Berker, Bristol, UK James Q. Del Rosso, Henderson, NV, USA Nilton Di Chiacchio, São Paulo, Brazil Ncoza Dlova, Durban, South Africa Zoe Diana Draelos, High Point, NC, USA Boni Elewski, Birmingham, USA Gabriella Fabbrocini, Naples, Italy Harold Farber, Philadelphia, PA, USA Steven Feldman, Winston-Salem, NC, USA

Stamatios Gregoriou, Athens, Greece Ramon Grimalt, Sant Cugat del Vallès, Spain

Daniela Guzman-Sanchez, Guadalajara, Mexico

Eckart Haneke, Freiburg, Germany Matilde Iorizzo, Bellinzona, Switzerland Akira Ishiko, Tokyo, Japan

Nathaniel J. Jellinek, East Greenwich, RI, USA
Ana Kaminsky, Buenos Aires, Argentina Alexandros Katoulis, Athens, Greece Yanna Kelly, São Paulo, Brazil Bryan Markinson, New York, NY, USA Amy McMichael, Winston-Salem, NC, USA

Andrew G. Messenger, Sheffield, UK Giuseppe Micali, Catania, Italy Mariya Miteva, Miami, FL, USA Bianca Maria Piraccini, Bologna, Italy Rodrigo Pirmez, Rio de Janeiro, Brazil Phoebe Rich, Portland, USA Bertrand Richert, Brussels, Belgium Adam I. Rubin, Philadelphia, PA, USA Lidia Rudnicka, Warsaw, Poland Julio Cesar Salas-Alanis,

San Pedro Garza Garcia, Mexico Richard K. Scher, New York, NY, USA Avner Shemer, Tel Hashomer, Tel-Aviv, Israel

Rodney Sinclair, East Melbourne, VIC, Australia

Michela Starace, Bologna, Italy Catherine Stefanato, London, UK Ralph M. Trüeb, Wallisellen, Switzerland Stefano Veraldi, Milan, Italy Colombina Vincenzi, Bologna, Italy Uwe Wollina, Dresden, Germany Martin Zaiac, Miami Beach, FL, USA 